\title{
The Do-It-Yourself Artificial Pancreas: A Comprehensive Review
}

Jothydev Kesavadev - Seshadhri Srinivasan • Banshi Saboo •

Meera Krishna B · Gopika Krishnan

Received: March 13, 2020 / Published online: April 30, 2020

(C) The Author(s) 2020

\begin{abstract}
Diabetes technology (DT) has accomplished tremendous progress in the past decades, aiming to convert these technologies as viable treatment options for the benefit of patients with diabetes (PWD). Despite the advances, PWD face multiple challenges with the efficient management of type 1 diabetes. Most of the promising and innovative technological developments are not accessible to a larger proportion of PWD. The slow pace of development and commercialization, overpricing, and lack of peer support are few such factors leading to inequitable access to the innovations in DT. Highly motivated and tech-savvy members of the diabetes community have therefore come
\end{abstract}

Digital Features To view digital features for this article go to https://doi.org/10.6084/m9.figshare.12145824.

J. Kesavadev $(\bowtie) \cdot$ M. Krishna B · G. Krishnan Jothydev's Diabetes Research Centre,

Mudavanmugal, Thiruvananthapuram, Kerala, India e-mail: jothydev@gmail.com

\section{S. Srinivasan}

Kalasalingam Academy of Research and Education, Srivilliputtur, Tamil Nadu, India

B. Saboo

DiaCare, Ahmedabad, Gujarat, India up with the \#WeAreNotWaiting movement and started developing their own do-it-yourself artificial pancreas systems (DIYAPS) integrating continuous glucose monitoring (CGM), insulin pumps, and smartphone technology to run openly shared algorithms to achieve appreciable glycemic control and quality of life (QoL). These systems use tailor-made interventions to achieve automated insulin delivery (AID) and are not commercialized or regulated. Online social network megatrends such as GitHub, CGM in the Cloud, and Twitter have been providing platforms to share these open source technologies and user experiences. Observational studies, anecdotal evidence, and realworld patient stories revealed significant improvements in time in range (TIR), time in hypoglycemia (TIHypo), HbA1c levels, and QoL after the initiation of DIYAPS. But this unregulated do-it-yourself (DIY) approach is perceived with great circumspection by healthcare professionals (HCP), regulatory bodies, and device manufacturers, making users the ultimate riskbearers. The use of the regularized CGM and insulin pump with unauthorized algorithms makes them off-label and has been a matter of great concern. Besides these, lack of safety data, funding or insurance coverage, ethical, and legal issues are roadblocks to the unanimous acceptance of these systems among patients with type 1 diabetes (T1D). A multi-agency approach is necessary to evaluate the risks, and to delineate the incumbency and liability of 
clinicians, regulatory bodies, and manufacturers associated with the use of DIYAPS. Understanding the potential of DIYAPS as the need of the present time, many regional and international agencies have come with strategies to appraise its safety as well as to support education and training on its use. Here we provide a comprehensive description of the DIYAPS-including their origin, existing literature, advantages, and disadvantages that can help the industry leaders, clinicians, and PWD to make the best use of these systems.

Keywords: AndroidAPS; Closed loop; Do-ityourself artificial pancreas; Loop; Nightscout; OpenAPS; People with diabetes; Type 1 diabetes; \#WeAreNotWaiting

\section{Key Summary Points}

Innovations in diabetes technologies are slow-paced, unaffordable, and unevenly distributed.

Frustrated diabetes community initiated \#WeAreNotWaiting movement and started developing do-it-yourself artificial pancreas systems (DIYAPS).

Comprehensive review of the current literature, advantages, and disadvantages of the DIYAPS is much needed.

History and evolution of DIYAPS, up-todate literature, potential benefits, and concerns about the system are presented in the review.

Patient experiences showed that DIYAPS resulted in significant improvements in QoL and TIR not achievable with the other approved devices. Yet, there are apprehensions about its safety and accountability.

This review will enable physicians to make an independent decision on the use of DIYAPS.

\section{INTRODUCTION}

Type 1 diabetes mellitus (T1D) is a chronic disease that must be managed over a life span. Multiple challenges and onset of complications due to ongoing hyperglycemia and hypoglycemia induced by absolute insulin deficiency and impaired counterregulatory hormone response result in poor quality of life, mood swings, and reduced life span in a significant number of patients with T1D [1]. Thus, there is an increased requirement for intensified insulin treatment regimens in patients with T1D in order to attain stringent glycemic control and to avert vascular complications. However, accomplishing this goal and preventing ketoacidosis and severe hypoglycemia is near to impossible with the conventional tools. Multiple daily injections (MDI) with long-acting insulin and short/rapid-acting insulin or via continuous subcutaneous insulin infusion (CSII) (insulin pump therapy) aided by frequent measurements of blood glucose either by selfmonitoring (SMBG) or by continuous glucose monitoring (CGM) have been widely adopted for better management of type 1 diabetes/brittle type 2 diabetes typically by patients in the developed world [2]. Despite these strategies, only $17 \%$ of youth and $21 \%$ of adult patients with T1D are reported to attain a target glycated hemoglobin (HbA1c) level of less than 7.0\% $(58 \mathrm{mmol} / \mathrm{mol})$, as recommended in clinical guidelines $[1,2]$. Daily self-management of T1D is a difficult assignment demanding vigorous planning and scrupulous execution of tasks for patients with diabetes (PWD) and their families $[3,4]$. Therefore, the administration of the correct amount of insulin at the right time still presents a great challenge for T1D self-management. Since T1D affects far fewer individuals, it is largely overlooked by governments and policy makers [5]. Considering these barriers to effective T1D self-management, PWD often strive for better tools and technologies.

The emergence of advanced and userfriendly technologies such as the new-generation glucose sensors and insulin pumps offers promising prospects to patient self-care through empowerment [6]. These technologies allow 
people with T1D and caregivers to precisely and constantly measure their blood glucose (BG) concentrations and regulate insulin infusion. But the high cost of closed loop systems, connectivity issues, automation-related errors, pump glitches, infusion site complications, etc. associated with insulin pumps were perceived as drawbacks by the T1D community [2, 7].

In 2006, CSII and CGM were combined to form the sensor-augmented pump therapy (SAP) [8]. The initial Medtronic systems gave only hypo and hyper alerts whereas the Veo and 530G Minimed insulin pumps could suspend insulin delivery at the onset of hypoglycemia. At present Minimed 670G, Tandem Control IQ, and Diabeloop are the commercially available hybrid closed loop systems. But technology snags and overpricing of these systems hinder their outreach. The research teams involved in the AP system development are currently at phase 3 or earlier phases of clinical trials. Moreover, regulatory procedures on the commercialization of the AP systems are very complex and time-consuming. Altogether, indications are that the T1D community has to wait for a long time for the next commercially viable products.

Frustrated with the slow pace and unaffordability of innovations in diabetes technology (DT), PWD have taken the responsibility of fasttrack development in their own hands. In 2013, PWD and their families/caregivers gathered online under the hashtag \#WeAreNotWaiting to share knowledge of the open source hardware and software solutions. Monitoring CGM data from a smartwatch, remotely tracking kids' BG levels, integration of louder alerts, and treatment suggestions based on the CGM data analysis to achieve automated control of insulin delivery are exemplary patient-driven projects that emerged from the \#WeAreNotWaiting movement $[9,10]$. Another dimension of the initiative was that tech-savvy T1D enthusiasts started self-building their own closed loop systems for automated insulin delivery (AID), also known as do-it-yourself artificial pancreas systems (DIYAPS) or open source artificial pancreas technology [11].

DIYAPS comprise commercially approved insulin pumps and CGMs that are connected and remotely controlled by systems using open source algorithms. Apparently, DIYAPS could overcome the psychological and behavioral obstacles necessitating continuous engagement from the patients and/or family and caregivers in adjusting the insulin regimen in response to glucose values/diet and exercise. The use of DIYAPS in these patients is expected to allow them to live a normal life, free from the nuances of minute to minute disease management burden.

Real-life experiences from patients and caregivers, anecdotal data, and published reports from selected cohorts have highlighted the clinical benefits and reduction in self-management burden with DIYAPS [12]. But there is a dearth of high-quality evidence exploring DIYAPS outcomes in a real-world context. As of now, the clinical governance, regulatory, and medicolegal status of DIYAPS are undefined [13]. Most essentially, we should identify the potential risks to users, as well as the ethical viewpoint of healthcare professionals (HCP) on the use of licensed devices in an off-label fashion. Only a global collaborative approach can resolve these ethical, legal, and regulatory constraints and facilitate broader and more rapid absorption of DIYAPS in T1D management.

Needless to say, the popularity of the DIY movement has been unprecedented since its introduction. DIYAP systems have become a more powerful, yet concerning tool that allows PWD to have a good night's sleep and to wake up with their glucose level in the target range $[14,15]$. In this article, we explain the concept and technical components of a DIY artificial pancreas system, provide reasons for choosing it, and expand on the benefits, possibilities, and concerns of using DIYAPS. We aim to draw attention to the current literature on DIYAPS and how it influences patient outcomes and regulatory concerns. We performed a literature search via PubMed using the following terms: \#WeAreNotWaiting, AndroidAPS, artificial pancreas system, automated insulin delivery, do-it-yourself, looping, Nightscout, OpenAPS, open source, and type 1 diabetes (T1D). This article is based on previously conducted studies and does not contain any studies with human 
participants or animals performed by any of the authors.

\section{HISTORY AND CONCEPT OF DIYAPS}

\section{\#WeAreNotWaiting}

People affected by Type 1 diabetes have been waiting for an affordable and efficient solution for the management of this chronic disease for decades. Lack of accessible and actionable data, unaffordability of the current systems, and long timeline of medical device development cycles have led to general exasperation in the T1D community. In 2013, the first DiabetesMine D-Data ExChange gathering at Stanford University spotlighted the sentiments and frustrations of patients with T1D waiting for their needs to be addressed and the term \#WeAreNotWaiting was coined. This event marked the beginning of the DIYAPS movement [9]. DIYAPS movement has witnessed the involvement of robust online communities of PWD, caregivers, and families who are communicating via the internet, including diabetes support forums, groups that have progressively collected on social media, and technology platforms such as Nightscout, "CGM in the Cloud" Facebook group, Twitter, Slack channel, and GitHub [16]. Through a gradual and systematic method of assembling, merging, and processing data from patients' devices to deliver significant actionable information, there has been a surge in the propagation and convergence of DIY diabetes device-related projects. In 2014, Dana Lewis, Scott Leibrand, and Ben West launched the OpenAPS project, providing the instructions and outline of a DIY patient-built artificial pancreas system (APS). The open source version, also known as OpenAPS, was launched in 2015 [10]. As of January 31, 2020, more than 1776 PWD around the globe have implemented various configurations of DIYAPS [17].

\section{D-Dads: Chasing the Impossible}

D-Dads are fathers whose fatherhood has been challenged by T1D. Unsatisfied with the inconvenience and unpredictability of diabetes care, some D-dads thought "outside the box" to assuage the burden of diabetes management. Their remarkable contributions led to the do-ityourself initiative.

Dr. Edward R. Damiano, a professor of biomedical engineering at Boston University, was motivated to develop a bionic pancreas when his 11-month-old son, David was diagnosed with T1D. Frustrated with the absence of reliable technologies, he created a bionic pancreas with the help of physicians and researchers [18]. In 2019, the US Food and Drug Administration (FDA) conferred "breakthrough device designation" to the iLet bionic pancreas [19]. Bigfoot Biomedical is another important name in the field of artificial pancreas (AP) development. The company was established by a group of D-dads, headed by Bryan Mazlish, a Wall Street quantitative analyst, Jeffrey Brewer, a former CEO of the Juvenile Diabetes Research Foundation (JDRF), and Lane Desborough, a chief engineer at Medtronic. Bigfoot Biomedical has been developing its homemade closed loop, the Bigfoot smartloop ${ }^{\mathrm{TM}}$ system. The company acquired the FDA's expedited "breakthrough device designation" in 2017. Mazlish earlier made a do-it-yourself closed loop system for his son Sam and became the first family to use a closed loop system in their own day-to-day lives [20]. Tidepool, a non-profitable organization was started by the D-Dads Howard Look and Steve McCanne and has been creating a regulated loop version of DIYAPS [21].

Development of Nightscout Project, a do-ityourself (DIY) mobile technology system, is recognized as a cornerstone in the DIYAPS mission. This system was invented by John Costik, the father of a 4-year-old boy Evan with T1D, with the help of Lane Desborough. Costik created a computer code to hack his son's CGM data and to upload the glucose values to the cloud via a mobile phone. The code granted him real-time access for monitoring his son's CGM data via the web or a smartphone app. Later, he made the Nightscout website and a 
private Facebook group called "CGM in the Cloud" to make the code openly available to a large community $[16,22]$. Pete Schwamb, a software engineer, invented RileyLink, a translator device to interconnect the insulin pump and an iPhone, in an effort to obtain access to the insulin pump data of his 6-year-old daughter Riley. Later, he co-developed the iOS DIYAPS version "loop", with Nathan Racklyeft [23].

These D-Dads have been making significant contributions to turn the artificial pancreas dream into reality, while focusing on its equitable access and affordability.

\section{COMPONENTS OF A DIYAPS}

The basic components of a DIYAPS are:

1. A real-time CGM - the CGM data can be real-time or intermittent (converted into continuous data using Miao Miao or Blucon readers). There are apps like $\mathrm{xDrip}+$ for Android and Spike for iOS that receive data from the CGM transmitter/sensor and display the readings on a smartphone. These are a combination of a device and a software application. The apps use the device to read the CGM output data and then upload this data to Nightscout so that the data can be accessed on the internet via a computer/ laptop or smartphone or smartwatch [24].

2. An insulin pump-the pump should be "loopable" to be used in DIY systems. Older versions of Medtronic pumps (Minimed Paradigm models 512/712, 515/715, $522 / 722$, and older version of $523 / 723$ ), taking advantage of the radiofrequency protocol, are most commonly used for this purpose. At present, Insulet's Omnipod Systems like EROS and DASH with Bluetooth protocols are also used in DIYAP systems.

3. A minicomputer or smartphone app-a minicomputer or smartphone app with the open source algorithm for calculating the insulin dose. The apps work as a data hub and processor between different devices.

Currently, there are three different DIYAP systems based on the app which bring together the insulin pump, CGM, and insulin dosing algorithm (https://openaps.org, https://diyps. org, https://androidaps.readthedocs.io/en/latest/ EN/index.html, https://www.Loopdocs.com, https://loopkit.github.io/loopdocs/). The three main types of DIYAPS are discussed here.

\section{OpenAPS}

OpenAPS is a safe, powerful, and easily understandable system that intends to adjust insulin dosage to maintain the BG levels in the recommended range, overnight and between meals. The first OpenAPS, developed by Dana Lewis, Scott Leibrand, and Ben West, and the code written with the help of Chris Hannemann was on a Raspberry Pi computer and a communication stick to connect to an old Medtronic pump.

Generally, an OpenAPS comprises an insulin pump, a CGM system, and an algorithm running on a microcomputer. The algorithms used in OpenAPS are oref0 (OpenAPS Reference Design Zero), Adjusting for unexpected BG deviation, and Bolus snooze. In recent times, an "Advanced Meal Assist (AMA)" feature has been incorporated into the OpenAPS algorithm. AMA gives a profoundly adaptable algorithm for securely dosing insulin after meals, regardless of broadly differing meal types, and the high variations in rates of digestion between individuals, making it the most widely used postprandial insulin dosing algorithm. The ultimate purpose of the OpenAPS system is to completely automate insulin dosing in all situations. In that direction, an oref1 algorithm has been developed that utilizes small "supermicroboluses (SMB)" of insulin at mealtimes and ensures more rapid and secure insulin delivery in response to $\mathrm{BG}$ rises.

A safety-focused reference design, open source reference implementation code, and documentation required for the set up are available on GitHub for the use of any individual or device manufacturer. OpenAPS reads the CGM data every $5 \mathrm{~min}$ and queries the insulin pump every few minutes for recent settings and activities such as current and maximum basal rates, recent boluses, insulin on board (IOB), 
insulin sensitivity factor (ISF), carb ratio (CR), duration of insulin acting (DIA), and BG target/ range, etc. On the basis of the communication from the insulin pump, OpenAPS updates the bolus wizard calculation and decides upon whether to cancel or issue a temporary basal. OpenAPS accomplishes this function through a physical piece of hardware called a "rig" that executes a sequence of commands to collect the CGM data, runs it through OrefO, and performs the dose calculations based on the pump setting values. The system can advise on changes in insulin to carbohydrate ratios and ISF settings through either Autosens (checking back 8-24 h) or Autotune (check back either $24 \mathrm{~h}$ or a userspecified period). Although this was the first developed system, recent users have been opting for AndroidAPS which offers more combinations of compatible devices and in-warranty pumps.

\section{Loop}

Here, the algorithm, which is different from OpenAPS, runs on an iOS operating system. The Apple iPhone receives CGM data and communicates with the insulin pump via Bluetooth. The first loop was developed by Nate Racklyeft and a D-Dad, Pete Schwamb, in 2016. Loop makes use of a free application, Xcode, to convert the raw code into an iOS application and install on an iPhone. Loop documentation is available on GitHub and the builders need to register as Apple developers to install the necessary software. Every $5 \mathrm{~min}$, the loop makes a forecast using BG values from $30 \mathrm{~min}$ ago and incorporates between that value and the current glucose value to make adjustments in insulin dose and to provide bolus recommendations and temporary basal rates. The app communicates with a small translator device called RileyLink that enables interaction between the pump, iPhone, and CGM. It is about the size of a tic-tac box and needs to be carried with you at all times. In a loop system, the pump speaks through radio language and the iPhone speaks through Bluetooth, and RileyLink acts as a translator to loop these parts together (https:// loopkit.github.io/loopdocs/faqs/rileylink-faqs/).
The use of Nightscout is recommended by loop developers, especially for parents or caregivers who aim to remotely track the loop activities.

\section{AndroidAPS}

AndroidAPS is an open source app with all functionalities of OpenAPS but runs on Google Android smartphones. The smartphone receives data from a CGM and communicates with the insulin pump via Bluetooth. First AndroidAPS was developed in Europe by Milos Kozak and Adrian Tappe in 2017 and it operates with modern in-warranty pumps with Bluetooth capability. The algorithms used here are Oref0 and Oref1. The app is available in different versions specific to geographic locations and languages. The basic modules of the profile include basal rates (BR), ISF, CR, and DIA. AndroidAPS provides multiple possibilities for remote monitoring of adults and pediatric patients with T1D. Parents and caregivers of kids with T1D can check the relevant data using the NSClient app on their Android phone. Features like alarms using xDrip+ app in follower mode, remote monitoring and control with SMS commands, and remote profile switch and temperature targets through the NSClient app contribute to the kid-friendly utility of this system.

Table 1 summarizes the combinations of different devices in the current DIYAPS platforms.

\section{CURRENT LITERATURE ON DIYAPS}

A large number of published self-reported, retrospective, observational, and prospective studies have reported that impressive outcomes such as better glycemic endpoints in sleep, reduction in self-management efforts, and improvements in QoL are possible with the use of various types of DIY closed loop implementations.

Lewis et al. collected and analyzed self-reported outcomes of OpenAPS in 2016 and 2018. In the 2016 study, 18 users (out of 40 total) reported a decrease in HbA1c levels from $7.1 \%$ to $6.2 \%$ and a rise in TIR from $58 \%$ to $81 \%$. The 
Table 1 Compatible devices for the three different configurations of DIYAPS. https://openaps.readthedocs.io/en/latest/ docs/Gear\%20Up/CGM.html, https://loopkit.github.io/loopdocs/build/step3/, https://androidaps.readthedocs.io/en/ latest/EN/Hardware/pumps.html

\begin{tabular}{lllll}
\hline Software & User interface & Hardware & CGM/FGM & Pumps \\
\hline OpenAPS & Small & Linux & Dexcom G6 & Medtronic \\
& computer & microcomputer & Dexcom G5 & 512/712, 515/715, 522/722, 523/723 (with \\
& Pebble watch & Rig & Dexcom G4 & firmware 2.4A or lower), Veo 554/754 EU \\
& & & Platinum & \\
& & MiniMelease (with firmware 2.6A or lower)
\end{tabular}

study also showed that all patients and caregivers experienced noteworthy improvements in sleep quality and peace of mind [14]. Later in 2018, Lewis et al. conducted a retrospective cross-over analysis with 20 OpenAPS users and observed that OpenAPS initiation has resulted in meaningful improvement in BG (135.7 to $128.3 \mathrm{mg} / \mathrm{dL})$, TIR $(75.8 \%$ to $82.2 \%)$, HbA1c $(6.4 \%$ to $6.1 \%)$, and reduction in time spent high and low during the day and at night [25].
A Korean study on pediatric patients with T1D (mean age $11.9 \pm 6.9$ years) reported a significant reduction in hypoglycemia and improved glycemic control (HbA1c and TIR) with the use of OpenAPS [26]. Similarly, a significant reduction in HbA1c levels and reduced time in hypoglycemia associated with DIYAPS use were reported by a study group from Italy [27]. In consonance with these findings, a UK observational study concluded that OpenAPS initiation 
Table 2 Review of glycemic outcomes of DIYAPS as reported from the published studies

\begin{tabular}{|c|c|c|c|c|}
\hline \multirow{3}{*}{$\begin{array}{l}\begin{array}{l}\text { Authors and } \\
\text { year of } \\
\text { publication }\end{array} \\
\text { Lewis et al. } \\
2016\end{array}$} & \multirow{3}{*}{$\begin{array}{l}\text { Publication } \\
\text { type } \\
\begin{array}{c}\text { Conference } \\
\text { abstract }\end{array}\end{array}$} & \multirow{3}{*}{$\begin{array}{l}\text { Aims } \\
\text { To analyze the shared and self-reported } \\
\text { data and experiences from } 18 \text { DIYAPS } \\
\text { users }\end{array}$} & \multicolumn{2}{|c|}{ Glycemic outcomes $(P<0.05)$} \\
\hline & & & $\mathrm{HbAlc}$ & $\begin{array}{c}7.1 \%(\mathrm{SD} 0.8 \%) \mathrm{vs} \\
6.2 \%(\mathrm{SD} 0.5 \%)\end{array}$ \\
\hline & & & $\begin{array}{l}\text { \%TIR }(80-180 \mathrm{mg} / \\
\quad \mathrm{dL})\end{array}$ & $\begin{array}{l}58 \%(\text { SD } 14 \%) \text { vs } 81 \% \\
\quad(\text { SD } 8 \%)\end{array}$ \\
\hline \multirow{6}{*}{$\begin{array}{l}\text { Lewis et al. } \\
2018\end{array}$} & \multirow{6}{*}{$\begin{array}{l}\text { Conference } \\
\text { abstract }\end{array}$} & \multirow{6}{*}{$\begin{array}{l}\text { To conduct a retrospective cross-over } \\
\text { analysis with } 20 \text { OpenAPS users }\end{array}$} & BG & 135.7 to $128.3 \mathrm{mg} / \mathrm{dL}$ \\
\hline & & & $\mathrm{HbAlc}$ & $6.4 \%$ vs $6.1 \%$ \\
\hline & & & $\% \mathrm{TIR}$ & $75.8 \%$ vs $82.2 \%$ \\
\hline & & & $\begin{array}{l}\text { Overnight, } \mathrm{BG} \\
\text { time }<70\end{array}$ & $6.4 \%$ vs $4.2 \%$ \\
\hline & & & $\begin{array}{l}\text { Overnight } \\
\text { time }<50\end{array}$ & $2.3 \%$ vs $1.0 \%$ \\
\hline & & & $\begin{array}{l}\text { BG } \\
\qquad \text { excursions }>300\end{array}$ & $1.7 \%$ vs $0.35 \%$ \\
\hline \multirow[t]{4}{*}{$\begin{array}{l}\text { Choi et al. } \\
2018\end{array}$} & \multirow[t]{4}{*}{$\begin{array}{l}\text { Conference } \\
\text { abstract }\end{array}$} & \multirow{4}{*}{$\begin{array}{l}\text { To present the clinical experience of } 20 \\
\text { patients }(11.9 \pm 6.9 \text { years }) \text { using } \\
\text { OpenAPS }\end{array}$} & $\mathrm{HbAlc}$ & $\begin{array}{c}6.8 \pm 1.0 \% \text { vs } \\
6.3 \pm 0.7 \%\end{array}$ \\
\hline & & & $\% \mathrm{TIR}$ & $\begin{array}{c}70.1 \pm 16.4 \% \text { vs } \\
83.3 \pm 10.1 \%\end{array}$ \\
\hline & & & $\% \mathrm{TAR}$ & $\begin{array}{c}24.7 \pm 16.5 \% \text { vs } \\
13.3 \pm 9.4 \%\end{array}$ \\
\hline & & & $\% \mathrm{TBR}$ & $\begin{array}{c}5.1 \pm 3.3 \% \text { vs } \\
3.4 \pm 2.3 \%\end{array}$ \\
\hline \multirow[t]{2}{*}{$\begin{array}{l}\text { Provenzano } \\
\text { et al. } 2018\end{array}$} & \multirow[t]{2}{*}{$\begin{array}{l}\text { Conference } \\
\text { abstract }\end{array}$} & \multirow{2}{*}{$\begin{array}{l}\text { To understand if closing the loop with } \\
\text { OpenAPS is effective to improve the } \\
\text { glucose control in T1D }\end{array}$} & $\mathrm{HbAlc}$ & $\begin{array}{l}7.17 \pm 0.49 \% \text { vs } \\
6.61 \pm 0.47 \%\end{array}$ \\
\hline & & & \%ТIHуро & $\begin{array}{l}8.55 \pm 5.81 \% \text { vs } \\
2.48 \pm 1.16 \%\end{array}$ \\
\hline \multirow[t]{3}{*}{$\begin{array}{l}\text { Wilmot et al. } \\
2019\end{array}$} & \multirow[t]{3}{*}{$\begin{array}{l}\text { Conference } \\
\text { abstract }\end{array}$} & \multirow[t]{3}{*}{$\begin{array}{l}\text { To compare the glycemic outcomes } \\
\text { (HbAlc, TIR, and TBR) of } 30 \text { people on } \\
\text { CSII with either OpenAPS versus } \\
\text { FreeStyle Libre (FSL) flash glucose } \\
\text { monitor }\end{array}$} & $\mathrm{HbAlc}$ & $\begin{array}{l}\text { OpenAPS }(7.3 \pm 1.4 \% \\
\text { vs } 6.2 \pm 0.4 \%) \\
\text { FSL }(7.6 \pm 0.8 \% \text { vs } \\
7.2 \pm 0.6 \%)\end{array}$ \\
\hline & & & \%TIR & $\begin{array}{l}\text { OpenAPS vs FSL } \\
(83.6 \pm 7.2 \% \text { vs } \\
55.9 \pm 11.5 \%)\end{array}$ \\
\hline & & & $\% \mathrm{TBR}$ & $\begin{array}{l}\text { OpenAPS vs FSL } \\
(2.5 \pm 1.8 \% \text { vs } \\
5.7 \pm 4.7 \%)\end{array}$ \\
\hline
\end{tabular}


Table 2 continued

\begin{tabular}{llll}
\hline $\begin{array}{l}\text { Authors and } \\
\text { year of }\end{array}$ & $\begin{array}{l}\text { Publication } \\
\text { type }\end{array}$ & Aims & Glycemic outcomes $(P<0.05)$ \\
publication & & & \\
\hline
\end{tabular}

Braune et al. Short paper 2019 2019

Melmer et al. Brief report

Koutsovasilis

et al. 2019
To conduct an online survey to assess the self-reported clinical outcomes of a pediatric population (median age 10 years) using DIYAPS in the real world

To analyze CGM records of 80 patients with T1D using DIY closed loop systems and to compare the glycemic outcomes of SAP therapy to OpenAPS in 34 of the users
HbAlc

$\%$ TIR

Glycemic parameters of DIY closed loop system users

$\begin{array}{ll}\text { Mean BG } & 137 \pm 20 \mathrm{mg} / \mathrm{dL} \\ \text { eAlc } & 6.40 \pm 0.70 \% \\ \text { TIR } & 77.5 \pm 10.5 \% \\ \text { TBR }<70 \mathrm{mg} / \mathrm{dL} & 4.3 \% \\ \mathrm{TBR}<54 \mathrm{mg} / \mathrm{dL} & 1.3 \% \\ \mathrm{TAR}>180 \mathrm{mg} / \mathrm{dL} & 18.2 \% \\ \text { TAR }>250 \mathrm{mg} / \mathrm{dL} & 4.1 \% \\ \text { SAP vs OpenAPS } & \end{array}$

\begin{tabular}{lc} 
Reduction in BG & $-0.6 \pm 0.7$ \\
Reduction in eAlc & $-0.4 \pm 0.5 \%$ \\
Increase in \%TIR & $+9.3 \pm 9.5 \%$ \\
3.9-10 mmol/L & \\
Reduction in & $-0.7 \pm 2.2 \%$ \\
TBR $<3.0 \mathrm{mmol} / \mathrm{L}$ & \\
Reduction in CV & $-2.4 \pm 5.8$ \\
Reduction in mean of & $-0.6 \pm 0.9 \mathrm{mmol} /$ \\
daily differences & $\mathrm{L}$ \\
HbAlc & $6.63 \pm 1.05 \mathrm{vs}$ \\
& $7.70 \pm 1.14$ \\
BG & $154.14 \pm 26.17 \mathrm{vs}$ \\
& $117.74 \pm 8.73$ \\
\hline
\end{tabular}

$6.91 \%$ [SD $0.88 \%)$ to 6.27\% [SD 0.67]

$64.2 \%$ [SD 15.94] to

$80.68 \%$ [SD 9.26]

$137 \pm 20 \mathrm{mg} / \mathrm{dL}$

$6.40 \pm 0.70 \%$

$77.5 \pm 10.5 \%$

TAR $>250 \mathrm{mg} / \mathrm{dL} \quad 4.1 \%$

SAP vs OpenAPS 
Table 2 continued

\begin{tabular}{|c|c|c|c|c|}
\hline $\begin{array}{l}\text { Authors and } \\
\text { year of } \\
\text { publication }\end{array}$ & $\begin{array}{l}\text { Publication } \\
\text { type }\end{array}$ & Aims & Glycemic outcomes (1 & $<0.05)$ \\
\hline \multirow{3}{*}{$\begin{array}{l}\text { Bazdraska } \\
\text { et al. } 2020\end{array}$} & \multirow{3}{*}{$\begin{array}{c}\text { Conference } \\
\text { abstract }\end{array}$} & \multirow{3}{*}{$\begin{array}{l}\text { To evaluate the benefits in children/ } \\
\text { adolescents using DIY unapproved loops } \\
\text { vs SAP therapy }\end{array}$} & \multicolumn{2}{|c|}{ DIY loop vs SAP therapy } \\
\hline & & & TIR & $83 \%$ vs $68.8 \%$ \\
\hline & & & $\begin{array}{l}\text { TIHypo }>14 \mathrm{mmol} / \\
\mathrm{L}\end{array}$ & $2.1 \%$ vs $8.6 \%$ \\
\hline
\end{tabular}

Glycemic outcomes are either reported as changes from baseline or comparison to another intervention

$B G$ blood glucose, $C S I I$ continuous subcutaneous insulin infusion, $C V$ coefficient of variation, DIY do it yourself, eAIc estimated HbAlc, SAP sensor-augmented pump, TAR time above range, TBR time below range, TIHypo time in hypoglycemia, $T I R$ time in range

in nine adult patients with T1D produced appreciable reduction of $\mathrm{HbA1c}(6.2 \pm 0.4$ vs $7.3 \pm 1.4 \%)$, increase in TIR $(83.6 \pm 7.2$ vs $55.9 \pm 11.5 \%)$, and reduction in time below range (TBR) $(2.5 \pm 1.8$ vs $5.7 \pm 4.7 \%)$ when compared with 30 patients using a continuous subcutaneous insulin infusion/FreeStyle Libre (FSL) flash glucose monitor (CSII/FSL) [28]. Litchman et al. using a qualitative netnography (Twitter analysis) method assembled responses on OpenAPS use from a larger population including patients, caregivers, and care partners $(n=328)$. The study suggested improvements in HbA1c, day-to-day glucose levels, and QoL. The study also reported that the patients perceived OpenAPS as a safe strategy [29].

In 2019, Braune et al. assessed the self-reported clinical outcomes of a pediatric population (age range 3-20 years) using DIYAPS through an online survey of the respective caregivers $(n=209)$. The study provided newage evidence on significant improvements in HbA1c levels (6.91\% to 6.27\%) and TIR (64.2\% to $80.68 \%$ ) in children and adolescents [30]. Melmer et al. analyzed CGM records of 80 patients with T1D (mean duration of 134 days per patient) and demonstrated the potential of DIY closed loop systems to produce stable glycemic outcomes [mean BG $137 \pm 20 \mathrm{mg} / \mathrm{dL}, \quad$ eA1c $6.40 \pm 0.70 \%, \quad$ TIR $77.5 \pm 10.5 \%, \quad$ TBR $<70 \mathrm{mg} / \mathrm{dL} \quad 4.3 \%$, TBR $<54 \mathrm{mg} / \mathrm{dL} 1.3 \%$, time above range $(\mathrm{TAR})>180 \mathrm{mg} / \mathrm{dL} \quad 18.2 \%, \quad$ TAR $>250 \mathrm{mg} / \mathrm{dL}$ $4.1 \%]$. The authors also observed that a change from sensor-augmented pump (SAP) therapy to OpenAPS in 34 of the users significantly reduced the mean glucose, estimated HbA1c, time in hypo and hyperglycemia, and accretion in TIR. In short, this study conclusively proved that OpenAPS systems are as competent as the other meticulously developed and tested devices [31, 32]. Koutsovasilis et al. reported that the use of OpenAPS systems in patients with T1D $(n=24)$ resulted in appreciable glycemic control in terms of $\mathrm{HbA} 1 \mathrm{c}$, fasting blood glucose (FBG), pre-meal, post-meal, and before bedtime glucose, less or no hypoglycemic events, and reduction in daily BG fluctuations [33]. In line with these results, a study from Bulgaria testified that the use of DIY loops significantly improved the TIR ( $83 \%$ vs $68.8 \%)$, time in hyperglycemia $>250 \mathrm{mg} / \mathrm{dL}$ (2.1 vs $8.6 \%)$, and HbA1c $(6.5 \%$ vs $7.2 \%)$ in T1D children $(n=31)$ [34]. In short, experiences shared by patients have shown improvements in quality of life with more TIR far above currently possible with approved devices. Table 2 summarizes the glycemic outcomes from acheived through DIYAPS as reported from the published studies.

An attempt to compare the efficiency of AndroidAPS and Loop configurations was made by Lal et al. in pigs with streptozocin-induced diabetes. The group observed that for unannounced meals, a better TIR was achieved with AndroidAPS than with Loop [35].

Petruzelkova et al. reported that the use of AndroidAPS among children with T1D (age $6-15$ years, $n=22$ ) participating in a 3-day 
winter ski camp resulted in a significant reduction in the BG levels, without any hypoglycemia episode. The study affirmed the safety, feasibility, and efficacy of AndroidAPS over SmartGuard ${ }^{\circledR}$ technology during and after prolonged physical activity [36]. In 2019, an in silico study using UVA/Padova Type 1 Diabetes Simulator proved that the AndroidAPS algorithm works safely and effectively and demonstrates great potential for use in home settings [37]. A most recent study has explained how a DIYAPS supported a woman with T1D to achieve two subsequent pregnancies [38]. These results are promising, but not sufficient to draw a conclusion about the safety and efficacy of DIYAP systems.

The Jaeb Center for Health Care Research (Tampa, FL, USA) is piloting a "Loop observational study" to evaluate the use of iOS-APS among patients with T1D. The study aims to collect information on loop operation, performance, major challenges, and the frequency of T1D complications such as severe hypoglycemia and diabetic ketoacidosis. This study is expected to provide a meaningful evaluation of the performance, safety, and patient-reported outcomes (PRO) of loop systems in T1D management [39]. Likewise, CREATE (Community deRivEd AutomaTEd insulin delivery) Trial, the first randomized controlled trial to assess the safety and efficacy of an AndroidAPS paired with a DANA-I insulin pump (SOOIL) and Dexcom G6 over SAP therapy in patients with T1D (aged 7-70 years), is scheduled to commence in due course [40].

Altogether these studies revealed that the potential of open source AP systems to attain stable glycemic control is comparable to other commercially developed technologies. The users identified several positive outcomes associated with the use of open source APS systems including increased TIR, reduced glucose variability, flexibility in food control, reduced hypoglycemia events, improved overnight glucose control, reduced nighttime hypo events, better sleep, and alleviated self-management/ disease burden. Meanwhile, one cannot rule out a likely bias in these findings and interpretations, as the participant cohorts included selected, technically adept, motivated users who were keen to manage the disease conditions by themselves. Therefore, it is imperative to understand whether similar results are achievable in the hands of users who are not so tech-savvy. The small sample size and short duration of these studies should also be considered as major limitations. Future studies should investigate the safety and efficiency of DIYAPS and its impact on the macro- and microvascular complications in T1D as well.

\section{WHY WOULD YOU OPT FOR AN OPEN SOURCE AP SYSTEM?}

\section{Potential Benefits}

The intention of open source artificial pancreas technology go beyond any commercial incentives, as it is conceived, designed, implemented, and supported by the global T1D community volunteers. The algorithms and apps are freely available for anyone with access to the internet.

The existing clinical-based evidence revealed incredible benefits such as better glycemic control, reduction in hypo and hyperglycemia events, and appreciable improvements in QoL related to DIYAPS use that serve as the motivational factors to adopt these innovative solutions. Users of these systems have pronounced the transition to DIYAPS from other technologies as "life-changing". The Open Project, backed by the European Union (EU), is conducting research involving a survey on the motivations, impediments, and maintenance of DIYAPS in the real world to enable a wider acceptance of these systems [41].

\section{Online Peer Support from the Loopers Community}

With more and more people building their own closed loop systems, the loopers community is equipped with 24 -h, global online support and makes consistent efforts to increase its radical transparency and accountability. The source codes of algorithms, reference design, online tutorials on how to integrate the algorithm with existing technology, setup procedures, and 
troubleshooting are available on the internet in plain language to explain the safety design and restrictions of DIY closed loop systems. This approach aims to aid the patients to make an informed decision and to make the right choice on DIYAPS [10].

An online survey reported that the patients and caregiver members of the "CGM in the Cloud" Facebook group have received and provided technical, emotional, and medical support through the online platform [22]. Patient stories and feedback are available at sites specific for different configurations of DIYAPS: https:// openaps.org, https://androidaps.readthedocs. io/en/latest/EN/, https://loopkit.github.io/ loopdocs/. The online loopers community provides commendable support for the selection, setup, and configuration of the devices so that the patients will not get discouraged by the complex nature of this technology [11]. These online communities also help in connecting people in person irrespective of geographical limitations [22]. Worldwide, small groups of DIY loopers gather for "DIY build parties" to review checklists and to close the loop by themselves [42]. These meetings are regularly announced on Facebook and Twitter and draw a large number of participants. Less expert users can get the support of DIYAPS experts to build their systems through these sessions [43].

\section{Impact on Quality of Life}

In the self-reported outcome study by Lewis, the responders reported improvements in sleep quality, family dynamics, family communication, and reduced disease management/psychological burden, altogether leading to enhancements in QoL of the patients and family members [14]. Marshall et al. provided the clinical experiences shared by two adults with T1D, the parent of a child with T1D, and three physicians emphasizing the impressive metabolic and psychological benefits after closing the loop. A parent story underlined the exclusion of carbohydrate micromanagement and improved sleep quality as the key benefits of DIYAPS [11]. Real-life experiences of 886 DIYAPS users compiled through an online survey reported that the initiation of DIYAPS led to dramatic improvements in clinical and QoL outcomes and produced positive emotional impacts [44].

Daily management of T1D is a time-consuming and labor-intensive process. It has been estimated that a person with "well-managed" T1D must devote a substantial amount of his or her time in "managing the unmanageable" [10]. Looping functions more closely like the pancreas to make insulin delivery as physiological as possible and to ease the disease management through future predictions of the BG levels. It has been estimated that DIYAPS users can save up to 1 day per month in time, thus alleviating the self-management burden of the disease [43].

\section{DIYAPS IS FOR SAFETY}

DIY systems are designed with user safety as a priority, the rationale behind the codes and reference designs being openly sourced. The OpenAPS site (https://openaps.org/referencedesign/) says that the "OpenAPS system is open and transparent in how it works, and understandable not just by experts, but also by clinicians and end-users (patients)". OpenAPS Reference Design, a simple language document available on the internet, explains about the safety design and restraints of DIYAP systems [10].

The algorithms and software are continuously being updated and improved, spotting the constraints of the hardware and software and spotlighting safety; the reference design enunciates the principles behind managing the missing or incorrect CGM data and loss of contact with the pump. The system does not depend on a single CGM value to adjust for any events of calibration or compression lows or sensor dropout. For safety purposes, the system is devised to simply retreat to conventional insulin pump mode in case of a lack of communication with the pump due to power loss. Since OpenAPS is being executed on external hardware rather than a built-in insulin pump algorithm, it works on setting temporary basal rates to attain the safe target range for BG. If new CGM data suggests that the temporary 
basal data is no longer suitable, OpenAPS can annul the current basal rate and go back to the pre-planned basal rate or even make necessary calculations. By ensuring these safety measures, DIYAP systems make sure that the most suitable amount of insulin is infused to mitigate the BG, with minimal risk of hypoglycemia. Furthermore, DIYAPS is based on the fundamental diabetes math a patient would be doing manually, thereby making the fabrication more transparent, simple, rational, and practical [45].

Another safety attribute of the DIYAPS is that the users are fully aware of what to anticipate from the system, can scrutinize the course and performance of the system, and exploit the system in a user-tailored manner to treat their disease. The approach is made possible by using the same insulin dosage and dosing algorithms including the basal rates, ISF, DIA, and IC ratio as prescribed by the patient's HCP [46].

\section{FINANCIAL BENEFITS}

So far, no study has addressed the cost-effectiveness of DIYAPS. Since these systems are not commercialized or marketed, no one makes a financial gain out of these systems. Regulated, approved, and commercialized closed loop systems are overpriced and difficult to afford by self-funding. Compared to these devices DIYAP systems are more economical, which indicates that the absorption of these systems is most likely to flourish soon. Sometimes, the procurement of compatible devices and hardware may demand an additional amount of investment. But peer support from the loopers community can make available free or cheaper devices within the community [47]. In the UK, PWD can access the parts required for a DIYAPS through the National Health Service (NHS). Many individuals who cannot afford a real-time CGM are utilizing FreeStyle Libre flash glucose monitor with a Miao Miao to connect to a UKfunded insulin pump via Bluetooth [43]. It has been reported that the DIYAPS users have better glycemic control and low risk of hypoglycemia, thus compensating for the costs associated with physician visits, cost-intensive treatment for hypoglycemia, and hospitalization.
Furthermore, the initiation of open source AP systems has shown to palliate the disease management and psychological burden of the PWD and families. This will eventually lead to better work efficiency, endurance, and access to a wide range of professions. All these factors can be beneficial to PWD from an economic point of view, provided the patients are fully aware of the risks and liabilities of using a DIYAPS.

\section{WHAT FACTORS ARE LIMITING WIDER ACCEPTANCE OF DIYAPS?}

DIYAP systems are purely a patient-led initiative, where technologies are established by motivated PWD and caregivers circumventing the trials and regulatory procedures necessitated for approval and commercialization of medical devices. Ultimately the individuals who decide to opt for these systems should use them at their own risk. The users will not be extended any discernible signs of support from HCP or regulatory bodies, even though the loopers community supports and facilitates DIYAPS use to a great extent. Underlying this are ambiguous contours of responsibility, in an episode of undesired consequence, between legalized device manufacturers, unregulated loopers, unregulated device providers, $\mathrm{HCP}$, regulatory bodies, and the end-user adopting an unregulated system like DIYAPS. According to our perception, the major concerns that ensue once a user starts to use a DIY system and demand substantial considerations are (1) technical issues, (2) safety issues, and (3) regulatory and ethical concerns.

\section{Technical Issues}

Patient stories suggested that the procurement of compatible devices to initiate the DIYAPS is the most difficult part. The setting up of Nightscout and other algorithms is time-consuming and requires technical know-how. The configuration can be tricky and complicated which can discourage the patients from choosing these systems. The users need to be engaged, activated, and committed to allocate time to 
understand and set up the system. The technical proficiency needed can be gained via the support from the DIY community. Here technical support from the community is indispensable for the users, as it may not be obtainable from HCP, clinics, or device manufacturers $[11,44]$. Increased power consumption by the smartphone and insulin pump is another matter of concern.

There are certain design constraints demonstrated with the design of the OpenAPS oref0 algorithm. One such constraint is that the algorithm cannot release insulin boluses.

Even though DIYAP systems have efficiently tackled several of these constraints, these issues make HCP and PWD apprehensive over the technology [11, 48].

\section{Safety Concerns}

Lack of sufficient safety and feasibility studies: Relative lack of data on the DIYAP systems creates a major challenge for the clinicians to discuss the potential safety concerns. The major chunk of supporting evidence is coming from selected cohorts of motivated, tech-savvy DIY users, which can introduce skewness to the results. Generalizations are made from observational studies and surveys conducted with a small number of participants and patient-reported experiences. Further, it could be claimed that "real-life" facts are stronger than those congregated and monitored in a controlled environment [49]. Moreover, the lack of evidence base for clinical- and cost-effectiveness hinders the establishment of supportive policies and funding on the DIYAPS approach [13].

Use of out-of-warranty, older insulin pumps: DIY users opt to use old, out-of-warrantied Medtronic insulin pumps, because the security flaw in these pumps could be exploited for a DIY revolution. Hackers make use of the security flaw to reverse engineer the old Medtronic pumps using self-made algorithms to calculate the insulin doses [48]. The chance of insulin pump failure is very low when used by techsavvy users, but it can be the other way when less-expert individuals start managing the older and out-of-warranty pumps. Looping created an underground market that sells old and used insulin pumps; the unknown history of these pumps, unrecorded defects, and lack of accountability of the vendors magnify the jeopardy associated with the scenario. Nevertheless, the advent of AndroidAPS which can communicate with commercially available pumps such as the Dana RS may tackle this matter [43].

Cybersecurity problem: Since data from DIY systems are exported into the cloud, externally monitored and accessed, a high probability of them getting hacked exists. The hacked data can be exploited by someone to control the insulin pump and one's insulin delivery. But the same risk applies to any device which communicates at radiofrequency. The OpenAPS users can embrace multiple strategies such as personalizing the pump and CGM settings and monitoring your insulin delivery online to mitigate the risk in the unlikely event of hacking [47]. Besides, the loopers need to ensure that the data is protected, meaning that the data transmitted externally by DIYAP systems should be befittingly encrypted before transmission [49].

\section{Regulatory and Ethical Concerns}

DIYAP systems are unregulated and not validated, hence posing undefined legal and ethical contemplations for HCPs, device manufacturers, finance providers, caregivers, and PWD. Offlabel use of regulated medical devices, concerns of indemnity among HCP and insurance providers, and caregivers' status to make a treatment decision on their discretion have been matters of intense debate.

Open source AP systems articulate unauthorized algorithms with authorized CGM devices and insulin pumps for use in T1D care. The combination of regulated and unregulated devices for treatment purposes makes their use off-label. Off-label use of already regularized devices creates a major challenge for HCPs, device manufacturers, and regulatory bodies. Even if the HCPs can perceive the 
improvements or benefits the patients are gaining with DIYAP systems, its off-label nature prevents them from endorsing these systems. The off-label use of the DIYAPS components may also lead to the annulment of insulin pump insurance or travel insurance [43].

Most often clinicians, owing to professional commitment, consider themselves responsible for treatment decisions the patients make, even if those are not recommended or prescribed by them. A survey conducted in the UK to understand the HCPs' attitudes towards the practice of DIYAPS reported that $91 \%$ of the responders did not support the use of DIY systems as a treatment option. The major reasons quoted behind this attitude were regulatory constraints, limited knowledge about the systems, potential risks associated with the inappropriate use, and concerns about indemnity. However, the majority of the participants expressed their willingness to continue support for the patients already using the DIY systems [50]. Under these circumstances, HCPs can play an integral role in understanding, implementing, and guiding PWD on various aspects of DIYAPS technology and to accomplish the most ideal care from it [51].

Regulatory bodies are anticipated to provide a transparent and stern framework to assess the risks and to demarcate the roles and responsibilities of clinicians, manufacturers, and PWD associated with DIYAPS. Since these systems are not commercialized, they are not regulated by the FDA. But in response to a reported adverse event associated with DIYAPS use, the FDA has issued a strong warning against the use of unauthorized devices or algorithms which are not reviewed or approved for safety and efficacy by the agency. The agency advised that PWD and HCP should bring any such adverse events to the notice of MedWatch, the FDA Safety Information and Adverse Event Reporting program [52]. In reply, the online DIYAPS communities have urged the users to report these kinds of undesired events as and when they happen [53].

Understanding the rising number of DIYAPS users in the UK, the JDRF has released a positional statement stating that the it cannot endorse the off-label use of DIY tech systems; however, the foundation respects the rights of patients with T1D to adopt the best treatments that befit their condition, including the use of DIY technology [54]. In 2018, a legal assessment of DIYAP systems was carried out by the German Diabetes Association, reconnoitering the medical, criminal, and civil law. The association did not identify the assembly of a DIY system as a criminal offense. But it explicitly recommended that the users and HCP identify the potential risks associated with the improper and off-label use devices. The loopers and HCPs were cautioned to be aware that the transfer of selfbuild DIY systems or training people on DIYAPS use will subject them to legal liability consequences [13].

The role of regulatory bodies should be to explicate that any off-label use by PWD and HCP must be executed carefully following the manufacturer's instructions and specifications for device interoperability, as well as to entitle the responsibilities of the third party performing the off-label use, to ensure that they are making safe use of the advanced technologies.

\section{FUTURE DIRECTIONS: CHANGING THE MINDSET}

The number of patients with T1D using DIYAPS technologies to manage their T1D has been increasing over the years. Even though these systems have produced commendable outcomes, there are concerns over their ethical and legal grounds. Understanding the capabilities of these systems, many not-for-profit organizations have come in support, to broaden the evenhanded access to APS technologies in the future. Tidepool commenced a project, temporarily named "Tidepool Loop", in collaboration with Insulet and Dexcom in 2018. This venture aims to deliver an officially supported, FDA-regulated DIY loop to be available by an HCP prescription and downloaded via the iOS App Store [55, 56]. This mission has been supported by JDRF and Helmsley Charitable Trust as a part of JDRF's Open-Protocol Initiative [54].

Top-rated diabetes conferences such as the American Diabetes Association (ADA) conference and Advanced Technologies and 
Treatments for Diabetes (ATTD) have incorporated scientific sessions on open source technology to encourage the study groups and patients to share their experiences and findings on international platforms [57]. Along the same line, the Association of British Clinical Diabetologists in the UK is planning to conduct a national clinical audit on DIYAPS use. The outcomes of this large-scale observational study will deliver the much-needed objective data on the influence of DIYAP systems on glycemic control, QoL, and associated risks [43, 58]. An open and collaborative multi-stakeholder approach integrating patients, nurses, diabetologists, general practitioners, and payers has been established by the pan-European CLOSE EIT Health innovation consortium to speed up a broad implementation of open source APS in T1D care while striking reasonable risk and cost-benefit balances [59].

\section{CONCLUSION}

PWD have been testing and self-modifying diabetes technologies for decades to improve their QoL and long-term prospects. Do-it-yourself artificial pancreas is an outcome of the radically changing outlook of the PWD community towards the slow pace and inequitable distribution of innovations in T1D treatment. User-specific automation in insulin delivery has been demonstrated to produce commendable glycemic outcomes and extricate the T1D community from the intensive burden of disease management. Here, peer support through online communities plays a formidable part to glue the support beyond geographic boundaries. Currently, loopers account for a minority population, but the numbers are growing progressively. Nevertheless, the lack of systematic practice-oriented studies is considered to be the stumbling block to the wider acknowledgment of DIYAP systems. Future studies should be designed considering the fact that the widespread implementation, support, and funding of open source systems for PWD depend absolutely on data validating safety and effectiveness. It is unquestionable that these systems will not replace clinicians, but rather will complement their efforts in patient care. The capability of DIYAP systems is well portrayed, yet in real-world clinical setups we are presented with an option: either to ignore the zeal concerning the potential of DIYAPS in routine clinical practice or to clarify the risks, regulatory and accountability issues and support in the training, education, and experiments to understand these systems. Through this comprehensive review our aim is to educate the readers to make an independent opinion on DIYAP systems. Patients are recommended to contemplate the risks with using DIYAPS against the risks of not using these. These undefined challenges will eventually be conquered, but it should not take so long that it hinders the wider adoption of this mature technology. The progression of DIYAPS should be a collective responsibility that amalgamates the efforts of PWD, regulators, healthcare providers, and industry leaders to get the best out of this innovation culture.

\section{ACKNOWLEDGEMENTS}

Funding. No funding or sponsorship was received for the publication of this article.

Authorship. All named authors meet the International Committee of Medical Journal Editors (ICMJE) criteria for authorship for this manuscript, take responsibility for the integrity of the work as a whole, and have given final approval for the version to be published.

Disclosures. Jothydev Kesavadev, Seshadhri Srinivasan, Banshi Saboo, Meera Krishna B, and Gopika Krishnan have nothing to disclose.

Compliance with Ethics Guidelines. This article is based on previously conducted studies and does not contain any studies with human participants or animals performed by any of the authors.

Data Availability. Data sharing is not applicable to this article as no datasets were generated or analyzed during the current study. 
Open Access. This article is licensed under a Creative Commons Attribution-NonCommercial 4.0 International License, which permits any non-commercial use, sharing, adaptation, distribution and reproduction in any medium or format, as long as you give appropriate credit to the original author(s) and the source, provide a link to the Creative Commons licence, and indicate if changes were made. The images or other third party material in this article are included in the article's Creative Commons licence, unless indicated otherwise in a credit line to the material. If material is not included in the article's Creative Commons licence and your intended use is not permitted by statutory regulation or exceeds the permitted use, you will need to obtain permission directly from the copyright holder. To view a copy of this licence, visit http://creativecommons.org/licenses/by$\mathrm{nc} / 4.0 /$.

\section{REFERENCES}

1. Chiang JL, Kirkman MS, Laffel LMB, Peters AL, Type 1 Diabetes Sourcebook Authors. Type 1 diabetes through the life span: a position statement of the American Diabetes Association. Diabetes Care. Diabetes Care. 2014;37:2034-54. https://pubmed. ncbi.nlm.nih.gov/24935775.

2. Senn J-D, Fischli S, Slahor L, Schelbert S, Henzen C. Long-term effects of initiating continuous subcutaneous insulin infusion (CSII) and continuous glucose monitoring (CGM) in people with type 1 diabetes and unsatisfactory diabetes control. J Clin Med. 2019;8:E394. https://pubmed.ncbi.nlm.nih. gov/30901914.

3. Vloemans AF, Eilander MMA, Rotteveel J, et al. Youth with type 1 diabetes taking responsibility for self-management: the importance of executive functioning in achieving glycemic control. Diabetes Care. 2019;42:225-31. https://care. diabetesjournals.org/content/42/2/225.abstract.

4. Vallis M, Willaing I, Holt RIG. Emerging adulthood and type 1 diabetes: insights from the DAWN2 Study. Diabet Med. 2018;35:203-13.

5. Kesavadev J, Sadikot S, Saboo B, et al. Challenges in type 1 diabetes management in South East Asia: descriptive situational assessment. Indian J Endocrinol Metab. 2014;18:600-7. https://www.ijem.in/ article. $\operatorname{asp}$ ?issn=2230-8210.
6. Alcántara-Aragón V. Improving patient self-care using diabetes technologies. Ther Adv Endocrinol Metab. 2019;10:2042018818824215. https://www. ncbi.nlm.nih.gov/pubmed/30728941.

7. Kesavadev J, Das AK, Unnikrishnan R 1st, et al. Use of insulin pumps in India: suggested guidelines based on experience and cultural differences. Diabetes Technol Ther. 2010;12:823-31.

8. Schönauer M, Thomas A. Sensor-augmented pump therapy-on the way to artificial pancreas. Av en Diabetol. 2010;26:143-6. https://www.elsevier.es/esrevista-avances-diabetologia-326-articulo-sensoraugmented-pump-therapy-on-S1134323010630025.

9. Omer T. Empowered citizen 'health hackers' who are not waiting. BMC Med. 2016;14:118. https:// doi.org/10.1186/s12916-016-0670-y.

10. Lewis DM. Do-it-yourself artificial pancreas system and the OpenAPS movement. Endocrinol Metab Clin N Am. 2020;49:203-13.

11. Marshall DC, Holloway M, Korer M, Woodman J, Brackenridge A, Hussain S. Do-it-yourself artificial pancreas systems in type 1 diabetes: perspectives of two adult users, a caregiver and three physicians. Diabetes Ther. 2019;10:1553-644. https://doi.org/ 10.1007/s13300-019-00679-y.

12. Lewis D, Leibrand S. Real-world use of open source artificial pancreas systems. J Diabetes Sci Technol. 2016;10:1411. https://doi.org/10.1177/1932296816 665635.

13. Oliver N, Reddy M, Marriott C, Walker T, Heinemann L. Open source automated insulin delivery: addressing the challenge. NPJ Digit Med. 2019;2: 124. https://doi.org/10.1038/s41746-019-0202-1.

14. Lewis D. Real-world use of open source artificial pancreas systems-poster presented at american diabetes association scientific sessions. 2016. https://openaps.org/2016/06/11/real-world-useof-open-source-artificial-pancreas-systems-posterpresented-at-american-diabetes-association-scientificsessions/. Accessed Feb 19, 2020.

15. Litchman ML, Lewis D, Kelly LA, Gee PM. Twitter analysis of \#OpenAPS DIY artificial pancreas technology use suggests improved A1C and quality of life. J Diabetes Sci Technol. 2019;13:164-70. https://pubmed.ncbi.nlm.nih.gov/30198751.

16. White K, Gebremariam A, Lewis D, et al. Motivations for participation in an online social media community for diabetes. J Diabetes Sci Technol. 2018;12:712-8. https://www.ncbi.nlm.nih.gov/ pubmed/29310448. 
17. OPENAPS.ORG. OpenAPS Outcomes. OPENAPS. ORG. 2020. https://openaps.org/Outcomes/. Accessed Feb 20, 2020.

18. Idlebrook $\mathrm{C}$. The diabetes dads behind 3 type 1 breakthroughs. Insulin Nation. 2015. https:// insulinnation.com/research/the-diabetes-dads-beh ind-3-type-1-breakthroughs/. Accessed Mar 2, 2020.

19. JDRF. FDA Grants breakthrough device status: iLet bionic pancreas. 2019. https://www.jdrf.org/blog/ 2019/12/23/fda-grants-breakthrough-device-statusilet-bionic-pancreas/. Accessed Mar 2, 2020.

20. Hoskins Mike and Tenderich Amy. Artificial pancreas: what you should know. Healthline. 2019. https://www.healthline.com/diabetesmine/artifi cial-pancreas-what-you-should-know\#1. Accessed Mar 10, 2020.

21. Glu. An interview with Tidepool CEO Howard Look. glu. 2014. https://myglu.org/articles/aninterview-with-tidepool-ceo-howard-look. Accessed Mar 2, 2020.

22. Gavrila V, Garrity A, Hirschfeld E, Edwards B, Lee JM. Peer support through a diabetes social media community. J Diabetes Sci Technol. 2019;13:493-7. https://www.ncbi.nlm.nih.gov/pubmed/30600704.

23. Racklyeft N. The history of Loop and LoopKit reflecting on the past in celebration of version 1.0. Medium. 2016. https://medium.com/@loudnate/ the-history-of-loop-and-loopkit-59b3caf13805. Accessed Mar 2, 2020.

24. Kev. WeAreNotWaiting thanks to \#xDrip-introduction. Circles Blue. 2015. https://circles-of-blue. winchcombe.org/index.php/2015/01/11/weareno twaiting-thanks-to-dexdrip-introduction/. Accessed Feb 20, 2020.

25. Lewis DM, Swain RS, Donner TW. Improvements in A1C and time-in-range in DIY closed-loop (OpenAPS) users. Diabetes. 2018;67:352-OR. https:// diabetes.diabetesjournals.org/content/67/ Supplement_1/352-OR.abstract.

26. Choi SB, Hong ES, Noh YH. Open artificial pancreas system reduced hypoglycemia and improved glycemic control in patients with type 1 diabetes. Diabetes. 2018;67:964-P. https://diabetes.diabetes journals.org/content/67/Supplement_1/964-P.abstract.

27. Provenzano V, Guastamacchia E, Brancato D, et al. Closing the loop with OpenAPS in people with type 1 diabetes-experience from Italy. Diabetes. 2018;67:993-P. https://diabetes.diabetesjournals. org/content/67/Supplement_1/993-P.abstract.
28. Wilmot EG, Langeland L, Mclay A, Taylor N. 1067-P: open source artificial pancreas system (APS) vs. combination insulin pump with flash glucose monitoring in adults with type 1 diabetes: an observational study. Diabetes. 2019;68:1067-P. https://diabetes.diabetesjournals.org/content/68/ Supplement_1/1067-P.abstract.

29. Litchman ML, Lewis D, Kelly LA, Gee PM. Twitter analysis of \#OpenAPS DIY artificial pancreas technology use suggests improved A1C and quality of life. J Diabetes Sci Technol. SAGE Publications Inc; 2018;13:164-70. https://doi.org/10.1177/1932296 818795705 .

30. Braune K, O’Donnell S, Cleal B, et al. Real-world use of do-it-yourself artificial pancreas systems in children and adolescents with type 1 diabetes: online survey and analysis of self-reported clinical outcomes. JMIR Mhealth Uhealth. 2019;7:e14087. https://mhealth.jmir.org/2019/7/e14087/.

31. Melmer A, Zuger T, Lewis DM, Leibrand S, Stettler C, Laimer M. Glycaemic control in individuals with type 1 diabetes using an open source artificial pancreas system (OpenAPS). Diabetes Obes Metab. 2019;21:2333-7.

32. Melmer A, Züger T, Lewis DM, Leibrand SM, Laimer M. 76-OR: in-depth review of glycemic control and glycemic variability in people with type 1 diabetes using open source artificial pancreas systems. Diabetes. 2019;68:76-OR. https://diabetes.diabetes journals.org/content/68/Supplement_1/76-OR.abstract.

33. Koutsovasilis A, Sotiropoulos A, Antoniou A, Kordinas V, Papadaki D, Peppas T. 1065-P: the effect of a closed-loop insulin delivery system on glycemic control in type 1 diabetes. Diabetes. 2019;68:1065P. https://diabetes.diabetesjournals.org/content/ 68/Supplement_1/1065-P.abstract.

34. Bazdraska Y, Iotova V, Mladenov V, Boyadzhiev V, Stoicheva RGS. 447-advantages from "do-it-yourself" loops among children and adolescents at Varna's Diabetes Center. ATTD 2020 e poster. 2020. https://doi.org/10.1089/dia.2020.2525.abstracts. Accessed Feb 24, 2020.

35. Lal RA, Maikawa CL, Lewis D, Buckingham BA, Appel EA. 463-comparing DIY full closed-loop performance in pigs with streptozocin-induced diabetes. ATTD 2020 e poster. 2020. https://cslide. ctimeetingtech.com/attd2020/attendee/eposter/po ster/474. Accessed Feb 24, 2020.

36. Petruzelkova L, Soupal J, Plasova V, et al. Excellent glycemic control maintained by open-source hybrid closed-loop AndroidAPS during and after sustained physical activity. Diabetes Technol Ther. 2018;20:744-50. https://doi.org/10.1089/dia.2018. 0214. 
37. Toffanin C, Kozak M, Sumnik Z, Cobelli C, Petruzelkova L. In silico trials of an open-source Androidbased artificial pancreas: a new paradigm to test safety and efficacy of do-it-yourself systems. Diabetes Technol Ther. 2019;22:112-20. https://doi. org/10.1089/dia.2019.0375.

38. Schutz-Fuhrmann I, Schutz AK, Eichner M, Mader JK. Two subsequent pregnancies in a woman with type 1 diabetes: artificial pancreas was a gamechanger. J Diabetes Sci Technol. 2020. https://doi. org/10.1177/1932296820906219.

39. Jaeb Center for Health Research. Welcome to the loop observational study. ClinicalTrials.gov. 2019. https://www.clinicaltrials.gov/show/NCT0383890 0. Accessed Feb 20, 2020.

40. Burnside M, De Bock M, Williman J, et al. CREATE (COMMUNITY DERIVED AUTOMATED INSULIN DELIVERY): randomised trial comparing automated insulin delivery (AnyDANA-Loop) using opensource algorithm, with sensor augmented pump therapy in type 1 diabetes. Diabetes Technol Ther. 2020;A1-A250. https://doi.org/10.1089/dia.2020. 2525.abstracts.

41. Open-diabetes. The Open Project. Outcomes of patients' evidence with novel, do-it-yourself artificial pancreas technology. Open. 2020. https:// open-diabetes.eu/en/welcome/. Accessed Feb 22, 2020 .

42. Wicks P. Patient, study thyself. BMC Med. 2018;16: 217. https://doi.org/10.1186/s12916-018-1216-2.

43. Crabtree TSJ, McLay A, Wilmot EG. DIY artificial pancreas systems: here to stay? Pract Diabetes. 2019;36:63-8. https://doi.org/10.1002/pdi.2216.

44. Cleal B, Braune K, O'Donnell S, et al. 78-LB: detailing the experiences of people with diabetes using do-ityourself artificial pancreas systems-qualitative analysis of responses to open-ended items in an international survey. Diabetes. 2019;68:78-LB. https://diabetes.diabetesjournals.org/content/ 68/Supplement_1/78-LB.abstract.

45. Lewis D. History and perspective on DIY closed looping. J Diabetes Sci Technol. 2019;13:790-3. https://pubmed.ncbi.nlm.nih.gov/30348013

46. OPENAPS.ORG. OpenAPS Reference Design. OPENAPS.ORG. 2020. https://openaps.org/ reference-design/. Accessed date Feb 20,2020.

47. OPENAPS.ORG. \#WeAreNotWaiting to reduce the burden of type 1 diabetes. OPENAPS.ORG. 2020. https://openaps.org. Accessed Feb 20, 2020.

48. Diabettech. DIY looping and CGM-a critical cog in the machine. 2019. https://www.diabettech.com/ artificial-pancreas/diy-looping-and-cgm/. Accessed Feb 19, 2020.

49. Garrison LPJ, Neumann PJ, Erickson P, Marshall D, Mullins CD. Using real-world data for coverage and payment decisions: the ISPOR Real-World Data Task Force report. Value Health. 2007;10:326-35.

50. Crabtree TSJ, Choudhary P, Hammond P, Lumb A, McLay A, Wilmot EG. Health-care professional opinions of DIY artificial pancreas systems in the UK. Lancet Diabetes Endocrinol. 2020;8:186-7. https://doi.org/10.1016/S2213-8587(19)30417-6.

51. Jennings P, Hussain S. Do-it-yourself artificial pancreas systems: a review of the emerging evidence and insights for healthcare professionals. J Diabetes Sci Technol. 2019. https://doi.org/10.1177/1932 296819894296.

52. FDA. FDA Warns against the use of unauthorized devices for diabetes management. FDA. 2019. https:// www.fda.gov/news-events/press-announcements/ fda-warns-against-use-unauthorized-devices-diabetesmanagement. Accessed Feb 22, 2020.

53. Caffrey M. FDA issues warning on do-it-yourself artificial pancreas. AJMC. 2019. https://www.ajmc. $\mathrm{com} /$ newsroom/fda-issues-warning-on-do-it-yourselfartificial-pancreas. Accessed Feb 20, 2020.

54. JDRF. JDRF's UK position statement on type 1 diabetes 'DIY' technologies. 2019. https://jdrf.org.uk/ about-us/position-statements-reports/positionstatements/jdrfs-uk-position-statement-on-type1-diabetes-diy-technologies/. Accessed Feb 22, 2020.

55. Look H. Tidepool intends to deliver Loop as a supported, FDA-regulated mobile app in the App Store. Tidepool. 2018. https://www.tidepool.org/blog/ tidepool-delivering-loop. Accessed Feb 21, 2020.

56. Snider C. Tidepool Loop, one year in: a development update. Tidepool. 2020. https://www.tidep ool.org/blog/tidepool-loop-development-update. Accessed Feb 21, 2020.

57. de Bock M. The 'do it yourself' type 1 diabetes dilemma for medical practitioners. Intern Med J. 2019;49:559-61. https://doi.org/10.1111/imj.14286.

58. Association of Brtitish Clinical Diabetologists. Doit-yourself artificial pancreas system (DIY APS) audit. Diabetol. 2020. https://abcd.care/DIYAPS. Accessed Feb 24, 2020.

59. Scliess F. Do-it-yourself artificial pancreas systems (DIY APS). Profil. 2018. https://blog.profil.com/ blog/do-it-yourself-artificial-pancreas-systems. Accessed Feb 22, 2020. 